\title{
Abnormal prefrontal brain activation during a verbal fluency task in bipolar disorder patients with psychotic symptoms using multichannel NIRS
}

This article was published in the following Dove Press journal: Neuropsychiatric Disease and Treatment

\author{
Jing-Jing Sun ${ }^{1,2}$ \\ Xiao-Min Liu ${ }^{2}$ \\ Chen-Yu Shen ${ }^{2}$ \\ Kun Feng ${ }^{1,2}$ \\ Po-Zi Liu ${ }^{2}$
}

'School of Clinical Medicine, Medicine Institute, Tsinghua University, Beijing, China; ${ }^{2}$ Yu Quan Hospital, Clinical Neuroscience Institute, Tsinghua University, Beijing, China
Correspondence: Po-Zi Liu Yu Quan Hospital, Tsinghua University, No 5 Shijingshan Road, Beijing 100049, China

Tel +86 $1088257755 ; 8213$

Fax +86 I0 8825 7755; 6IIII

Email liupozi@mail.tsinghua.edu.cn
Purpose: Bipolar disorder (BD) patients with psychotic symptoms (BDp) worsens prognosis and decreases rates of recovery. The study investigated cognitive performance and brain function between BD patients in depressive episode with and without psychotic symptoms to find potential neurobiological markers associated with psychotic features of BD patients in depressive episode.

Patients and methods: Thirty-one patients without psychotic symptoms and 29 patients with psychotic symptoms diagnosed with bipolar I disorder with a current depressive episode were included in the present study. The neural function of prefrontal cortex (PFC) and cognitive performance among BDp, BD patients without psychotic symptoms, and 23 healthy controls (HC) were examined using near-infrared spectroscopy during verbal fluency task (VFT).

Results: 1) Both the BD groups exhibited significantly worse performance of VFT and lower activation of oxygenated hemoglobin (oxy-Hb) changes in the bilateral ventrolateral PFC compared with the HC group. 2) Within the BD group, VFT performance was not significantly different. 3) The prefrontal activation of oxy-Hb changes in the BDp patients was significantly lower than that in the BD patients without psychotic symptoms in the right dorsolateral PFC. 4) Activation of oxy-Hb changes in right dorsolateral PFC was negatively correlated with the severity of psychotic symptoms in BDp patients.

Conclusion: The prefrontal function differs between BD patients in depressive episode with or without psychotic symptoms measured with near-infrared spectroscopy.

Keywords: bipolar disorder, depressive, psychotic symptoms, near-infrared spectroscopy, verbal fluency task

\section{Introduction}

More than $50 \%$ of all patients with bipolar disorder (BD) present with psychotic symptoms on at least one occasion. ${ }^{1,2}$ Symptoms associated with psychosis worsen the patient's prognosis and decrease recovery rates, ${ }^{3,4}$ as well as possibly implicating which pharmacological treatment is used ${ }^{5,6}$ and affecting psychosocial functioning in BD. ${ }^{7}$ Studying potential neurobiological markers associated with psychotic features might help in furthering the understanding of the pathophysiology of this disease and improve its treatment, hence improving the prognosis.

With the development of neuroimaging technology, many studies have been conducted aimed at investigating structure, local blood flow, and neurotransmitter changes in the brain of patients diagnosed with BD. Gruber et $\mathrm{al}^{8}$ reported decreased cingulate cortex activation and increased prefrontal cortex (PFC) activation may be associated with impaired cognitive control in BD patients. Nishimura et $\mathrm{al}^{9}$ showed that 
BD patients exhibited significantly lower activation during the verbal fluency task (VFT) in the broader bilateral PFC. Accordingly, there are increasing reports, appearing in the literature, of neuroimaging studies carried out on BD patients who have been grouped according to whether they have or have not experienced psychotic symptoms. For example, Radaelli et al $^{10}$ studied 34 delusional and 39 non-delusional patients, diagnosed with BD, using structural magnetic resonance imaging (MRI). A reduction in the volume of gray matter (GM) in the dorsolateral prefrontal cortex (DLPFC) and insula was identified in delusional BD patients with delusional symptoms. When Anticevic et $\mathrm{al}^{11}$ studied BD patients with a history of psychosis, it was demonstrated that there was a distinct pattern of PFC disconnectivity. In another investigation into functional connectivity, BD patients with a history of psychosis showed significantly reduced ventral anterior cingulate cortex connectivity extending to the ventral and dorsal medial PFC, in comparison to patients with no history of psychotic events, but not significantly different from those found in patients suffering from schizophrenia. ${ }^{12}$ So, the results of previous studies suggest that it is reasonable to hypothesize that the function of the PFC differs in BD patients with psychotic symptoms (BDp) and BD patients without psychotic symptoms (BDn).

However, almost all researchers studying BD patients with a history of psychosis using imaging methods to observe brain region dysfunctionality did so during the resting state. So far, brain changes specifically related to psychosis have not linked the performance of cognitive tasks with neuroimaging results. Although there is a lot of evidence to demonstrate that cognitive impairment ${ }^{13-15}$ occurs in patients with BD, such as losses of attention, memory, and executive functioning, the specific effect of psychotic symptoms on cognitive functioning in BD remains unclear. Some studies have suggested that there is a greater cognitive impairment in BD patients with a history of psychotic symptoms than those who have not demonstrated psychotic symptoms. ${ }^{16,17}$ In other studies, no significant differences between BD patients with and without a history of psychotic symptoms could be demonstrated. ${ }^{18,19}$ These inconsistent results are unsurprising, as whether cognitive impairments remain relatively intact or patients continue presenting with cognitive dysfunction upon the remission of psychotic symptoms is unknown. In addition, patients studied in previous studies were free of psychotic symptoms at the time of examination, and it is doubtful whether PFC function would recover to normality or continue presenting dysfunctionally.

A new optical neuroimaging technique, functional near-infrared spectroscopy (NIRS), with the advantages of being low cost, relatively nonconstraining, noninvasive, and convenient is widely used to study neural activity in many psychiatric diseases (such as depression disorder and schizophrenia). Previous studies using NIRS have reported neural hypoactivity, ${ }^{20-22}$ hyperactivity, ${ }^{23}$ and differential time courses of activation ${ }^{24}$ during the cognitive tasks performed by BD patients. However, NIRS studies have rarely taken psychotic symptoms into consideration.

It is well known that psychotic symptoms are more common during a manic episode in BD patients. ${ }^{25}$ However, there are studies reporting that psychosis is frequent in bipolar depressive episodes. ${ }^{26}$ Fountoulakis et $\mathrm{al}^{27}$ recently reported that the prevalence of psychosis in bipolar depressive episodes can be as high as $66 \%$. Compared to psychotic manic episodes, BD with psychosis during depressive episodes is also associated with a more frequent history of suicidality. ${ }^{28}$ While there is a high prevalence and a possible impact of psychosis in bipolar depression, the differences between bipolar depression with psychotic symptoms and bipolar depression without psychotic symptoms have not been well studied. Epidemiologic studies indicate that BD patients have higher rates of substance abuse, anxiety disorders, and obsessive-compulsive disorders. ${ }^{29,30}$ Previous studies have suggested that the severity of substance abuse is negatively correlated with activation of the PFC and of the caudate in patients with BD. ${ }^{31}$ In another MRI/PET study, it was found that BD patients with a comorbidity of substance abuse demonstrated reductions in the volume of the ventrolateral PFC (VLPFC), anterior cingulate, insula, and thalamus. ${ }^{32}$ There are no studies on the co-occurrence of anxiety disorders and obsessive-compulsive disorders and how they interact in the bipolar brain. All we know is that using NIRS, patients with social anxiety disorder showed lower activation of oxygenated hemoglobin (oxy-Hb) concentrations in the VLPFC during a VFT. ${ }^{33}$ Compared with normal controls, patients diagnosed with obsessive-compulsive disorder exhibited reduced PFC hemodynamic responses during verbal fluency and Stroop color-word tasks. ${ }^{34,35}$ These studies suggest that the PFC plays an important role in the pathophysiology of substance abuse, anxiety disorders, and obsessive-compulsive disorders. We cannot conclude that the differences in PFC function result from psychotic symptoms in BD because psychiatric comorbidities were rarely controlled for. As a result, patients who had psychiatric disorders other than BD were excluded from the present study. VFT is a kind of task that is widely used in neuropsychological cognitive assessment. It can detect PFC activation combined with NIRS in healthy people and mental disorder patients ${ }^{36,37}$ due to the closed relationship between frontal brain area and performance in VFT. ${ }^{38}$ In addition, it 
is among the strongest findings of cognitive abnormalities in patients with BD. Therefore, VFT was selected as the cognitive task in this study.

The aim of the present study was to evaluate cognitive performance and the prefrontal hemodynamic response in the PFC area, using NIRS, in a depressive episode in BD patients with, or without, psychotic symptoms during VFT. Prior to the study, it was hypothesized that both groups of BD patients would demonstrate impaired performance and decreased prefrontal activation. It was further hypothesized that the prefrontal activation pattern would differ in BD patients with and without psychotic symptoms.

\section{Methods}

\section{Subjects}

Subjects were recruited from outpatient and inpatient departments of Yu Quan Hospital from February 2013 to February 2016. Patients without psychotic symptoms during the study ( $\mathrm{n}=31 ; \mathrm{BDn})$ and patients with psychotic symptoms $(\mathrm{n}=29 ; \mathrm{BDp})$ and diagnosed with bipolar I disorder with a current depressive episode were included in the present study. All subjects meeting bipolar I disorder diagnostic criteria were evaluated by experienced psychiatrists with fellowship training (Po-Zi Liu and Kun Feng) using the Structured Clinical Interview according to the Diagnostic and Statistical Manual of Mental Disorders, version 4. Twenty-three healthy controls (HC) were recruited from the local community via advertisements. Age, gender, educational level, and righthand dominance were matched between BD groups and control subjects. The study was approved by the ethics committee of Yu Quan Hospital and written, informed consent was obtained from all participants prior to the commencement of the study.

Patients with a history of psychosis were excluded from the present study. Participants were also excluded for the coexistence of any of the following conditions with BD: pregnancy; major physical illnesses such as malignant tumor, chronic renal failure, severe heart disease, and severe respiratory disease; other psychiatric disorders and neurological disorders; chronic substance abuse; and cognitive disorders. None of the patients had taken medication or received electroconvulsive therapy within the 6-month period prior to the study.

\section{VFT task}

This study took four-block (vegetables, domestic applications, four-legged animals, and fruits) semantic category versions of the VFT as the cognitive task. The VFT task was used to assess PFC activity ${ }^{39,40}$ by measuring the relative hemoglobin concentration changes. The total measurement time was 360 seconds and each block measurement was for 90 seconds, which included a 30 -second pre-task baseline, a 30-second VFT, and a 30-second post-task baseline (Figure 1). All the participants were instructed to sit quietly and stare at the screen, which was $50 \mathrm{~cm}$ in front of them, during the pre-task and post-task baseline. They were instructed to generate as many items as possible that belonged to the related species within the task period. The numbers of correct words generated during the task period were recorded to assign cognitive performance scores. The accuracy (\%) of the correct words was also used to evaluate behavioral performance.

\section{NIRS measurements}

An NIRS device (FOIRE-3000, Shimadzu Corporation, Kyoto, Japan) with 45 channels was used to measure three types of relative concentration change of hemoglobin (oxy-Hb, deoxygenated hemoglobin, and total hemoglobin) during the VFT task. Fourteen pairs of emission probes were placed on the forehead of each participant at distances

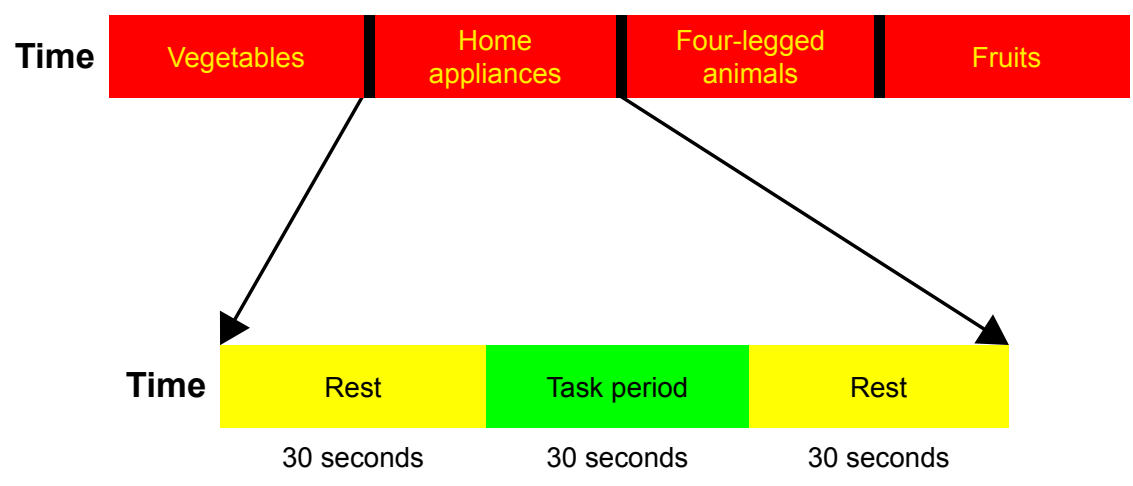

Figure I VFT task design.

Note: Four-block (vegetables, domestic applications, four-legged animals, and fruits) semantic category versions of VFT included a 30-second pre-task baseline, a 30-second VFT, and a 30-second post-task baseline. Abbreviation: VFT, verbal fluency task. 
of $3.0 \mathrm{~cm}$ apart. Each pair of emission-detector probes was defined as a "channel" at a measurement point. The NIRS probes covered the participant's frontal region. The most inferior probes were positioned 25-30 $\mathrm{mm}$ above the midpoint of the eyebrow of participants, which is, according to the international 10-20 system of electroencephalogram electrode placement, along the Fp1-Fp2 line. ${ }^{41}$

\section{Clinical assessments}

The 24-item Hamilton Depression Scale (HAMD) ${ }^{42}$ and the Young Mania Rating Scale (YMRS) ${ }^{43}$ were used to assess symptoms of depression and mania, respectively, in participants. The expanded version of the Brief Psychiatric Rating Scale (BPRS) ${ }^{44}$ was used to assess psychiatric symptomatology at the time of the investigation. Edinburgh handedness inventory was used to evaluate the handedness of participants. Demographic information was collected using questionnaires developed by our research group.

\section{Statistical analysis}

All statistical analyses were performed using the Statistical Package for the Social Sciences for Windows (SPSS version 22.0; IBM Corporation, Armonk, NY, USA). We used a one-way ANOVA to compare demographic and clinical variables, as well as VFT performances between the three groups, with the exception of gender (contingency table analysis). We also compared the three groups' oxy-Hb changes using ANOVA in order to analyze differences in prefrontal function. The value of oxy-Hb changes in each channel was calculated by subtracting the mean oxy-Hb in the task period from that in the pre-task period. Due to its superior signal to-noise ratio, oxy-Hb was selected as the statistical index ${ }^{45}$ and reliability indicator ${ }^{46,47}$ in order to reflect changes in regional cerebral blood flow. The least significant difference (LSD) was used to process the post hoc test on significant variables. Homogeneity of variance was tested to compare the distribution shape of the different groups. For multiple comparison analyses of 45-channel testing correction, Benjamini-Hochberg's procedure was adopted with a false discovery rate $(F D R=0.2)$ so that there are no more than $20 \%$ false positives on average (FDRcorrected). For the three groups, we calculated Spearman's rank correlation coefficients to examine the relationship between oxy-Hb changes during the VFT and the HAMD, YMRS, and BPRS scores for each channel.

\section{Results \\ Demographic characteristics and clinical symptoms}

The demographic characteristics of the three groups are shown in Table 1. Among the BDn, BDp, and HC groups, there were no significant differences in age, gender, educational level, and severity of hypomanic symptoms as assessed by the YMRS; HAMD scores were not significantly different

Table I Characteristics of subjects

\begin{tabular}{|c|c|c|c|c|c|c|c|}
\hline Demographics & BDn & BDp & $\mathrm{HC}$ & $\begin{array}{l}\text { Significance } \\
\text { (BDn, BDp, HC) }\end{array}$ & $\begin{array}{l}\text { Significance } \\
\text { (BDn vs HC) }\end{array}$ & $\begin{array}{l}\text { Significance } \\
\text { (BDp vs HC) }\end{array}$ & $\begin{array}{l}\text { Significance } \\
\text { (BDn vs BDp) }\end{array}$ \\
\hline Age (years) & $30.93 \pm 8.98$ & $28.38 \pm 6.83$ & $32.91 \pm 10.18$ & 0.173 & 0.410 & 0.065 & 0.257 \\
\hline Gender (female/male) & $17 / 14$ & $14 / 15$ & $11 / 12$ & 0.837 & & & \\
\hline Education level (years) & $|3.5| \pm 3.18$ & $13.82 \pm 3.06$ & $13.47 \pm 3.55$ & 0.908 & 0.966 & 0.710 & 0.711 \\
\hline YMRS & $1.48 \pm 1.6 \mathrm{I}$ & $1.17 \pm 1.44$ & $1.39 \pm 1.37$ & 0.334 & 0.822 & 0.600 & 0.421 \\
\hline HAMD & $24.7 I \pm 3.39$ & $25.24 \pm 3.59$ & $7.04 \pm 4.24$ & $<0.001$ & $<0.001$ & $<0.001$ & 0.581 \\
\hline BPRS & $26.83 \pm 4.65$ & $82.72 \pm 24.22$ & $26.60 \pm 5.03$ & $<0.001$ & 0.955 & $<0.001$ & $<0.001$ \\
\hline \multicolumn{8}{|l|}{ Vegetables } \\
\hline Corrected word & $7.77 \pm 2.26$ & $8.03 \pm 2.27$ & $10.04 \pm 2.72$ & 0.002 & 0.001 & 0.004 & 0.676 \\
\hline Accuracy & $90.34 \pm 10.05$ & $92.26 \pm 10.62$ & $91.80 \pm 6.75$ & 0.728 & 0.587 & 0.863 & 0.445 \\
\hline \multicolumn{8}{|l|}{ Family applications } \\
\hline Corrected word & $7.64 \pm I .52$ & $7.27 \pm 1.38$ & $9.43 \pm 1.78$ & $<0.001$ & $<0.001$ & $<0.001$ & 0.360 \\
\hline Accuracy & $86.94 \pm|I .4|$ & $88.7| \pm| 3.34$ & $88.02 \pm 8.02$ & 0.832 & 0.730 & 0.830 & 0.549 \\
\hline \multicolumn{8}{|l|}{ Four-foot animals } \\
\hline Corrected word & $7.09 \pm 1.66$ & $7.13 \pm 1.24$ & $7.71 \pm 1.69$ & $<0.001$ & $<0.001$ & $<0.001$ & 0.910 \\
\hline Accuracy & $88.73 \pm 11.43$ & $90.01 \pm 11.48$ & $93.48 \pm 7.56$ & 0.255 & 0.105 & 0.242 & 0.683 \\
\hline \multicolumn{8}{|l|}{ Fruits } \\
\hline Corrected word & $7.00 \pm 1.18$ & $7.62 \pm 2.62$ & $9.08 \pm \mid .31$ & $<0.001$ & $<0.001$ & 0.006 & 0.197 \\
\hline Accuracy & $85.01 \pm 11.99$ & $87.31 \pm 12.46$ & $88.65 \pm 8.96$ & 0.495 & 0.251 & 0.674 & 0.439 \\
\hline
\end{tabular}

Note: Data are expressed as mean \pm SD.

Abbreviations: BDn, BD patients without psychotic symptoms; BDp, BD patients with psychotic symptoms; BPRS, Brief Psychiatric Rating Scale; HAMD, Hamilton Rating Scale for Depression; HC, healthy controls; YMRS, Young Mania Rating Scale. 
between patients in the $\mathrm{BDn}$ group and $\mathrm{BDp}$ group, but there were significant differences between $\mathrm{BDp}$ and $\mathrm{HC}$ groups and between BDn and $\mathrm{HC}$ groups. BPRS scores between patients in the BDn group and $\mathrm{HC}$ were not significantly different, but there were significant differences between patients in the BDp and $\mathrm{HC}$ groups and between patients in the BDp and $\mathrm{BDn}$ groups.

\section{Performance of VFT task}

During the VFT, the number of words generated among the three groups showed statistically significant differences, as presented in Table 1. Both the BDn and BDp groups generated a significantly different number of words in all categories when compared with the HC group; the LSD was used to compare two of the three groups, and significant differences between $\mathrm{BDn}$ patients and $\mathrm{HC}$ groups were identified in all categories $(P<0.001)$. The $\mathrm{BDp}$ group and $\mathrm{HC}$ group were also shown to be significantly different in all categories ( $P=0.004, P<0.001, P<0.001, P=0.006$ ). Furthermore, no significant differences were determined in any of the four categories between the BDn and BDp groups $(P>0.05)$. The accuracy $(\%)$ of the corrected words among the three groups showed no significant differences, as shown in Table 1 $(P>0.05)$.

\section{Group comparisons of NIRS activation}

We compared the oxy-Hb changes among the three groups using ANOVA. Changes in oxy-Hb were homogeneous in 32 channels ( $P>0.05)$, except channels 5, 7-11, 19, 25, 26, $29,35,37,43$. In the left channels, channels $14,15,18,20$, $21,32,33,38-41$ showed significant differences among groups (FDR-corrected $P<0.2$ ).

\section{Post hoc test (LSD)}

The LSD was used to examine the post hoc analysis of covariance. We observed significant differences in oxy- $\mathrm{Hb}$ changes in channels 33, 38-40 (FDR-corrected $P<0.2$, Figure 2) between BDn patients and HC groups. The BDn patients exhibited significantly lower oxy-Hb activation than the HC group during the VFT task in four channels, located approximately in the bilateral VLPFC. These data suggest that there were significant differences in the oxy-Hb changes in channels 14, 15, 18, 20, 21, 32, 33, 38-41 (FDR-corrected $P<0.2$; Figure 3) between the BDp and HC groups. Patients in the $\mathrm{BDp}$ group exhibited significantly smaller amplitude oxy-Hb activation than the $\mathrm{HC}$ group during the VFT task in 11 channels, located approximately in the bilateral VLPFC and the bilateral DLPFC. Both BD groups showed a reduced

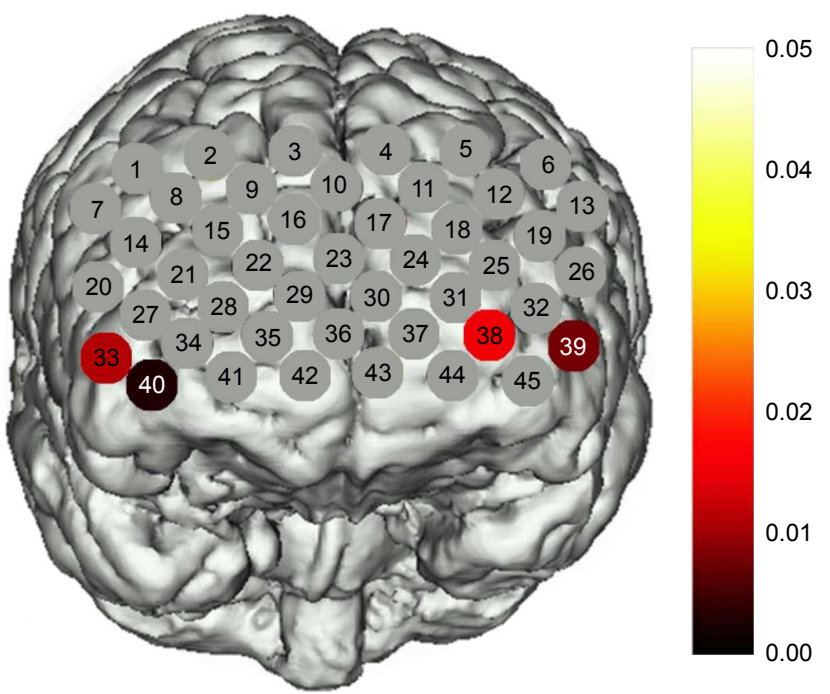

Figure 2 A map showing statistically significant lower oxy-Hb changes in BDn patients compared with the controls during the VFT.

Notes: Channels in color show significantly lower oxy-Hb changes during the VFT in $\mathrm{BDn}$ compared with healthy controls (FDR-corrected $P<0.2$ ). Channels in gray show FDR-corrected $P$-values $>0.2$.

Abbreviations: BDn, bipolar disorder patients absence of psychotic symptoms; FDR, false discovery rate; oxy-Hb, oxygenated hemoglobin; VFT, verbal fluency test.

activation compared with the $\mathrm{HC}$ group in channels 33 , 38-40, which were located approximately in the bilateral VLPFC (Figure 4). Within the two BD groups, the mean oxy-Hb changes in $\mathrm{BDp}$ patients were significantly lower than those in BDn patients in channel 14, which was located in the right DLPFC (FDR-corrected $P<0.2$; Figure 5).

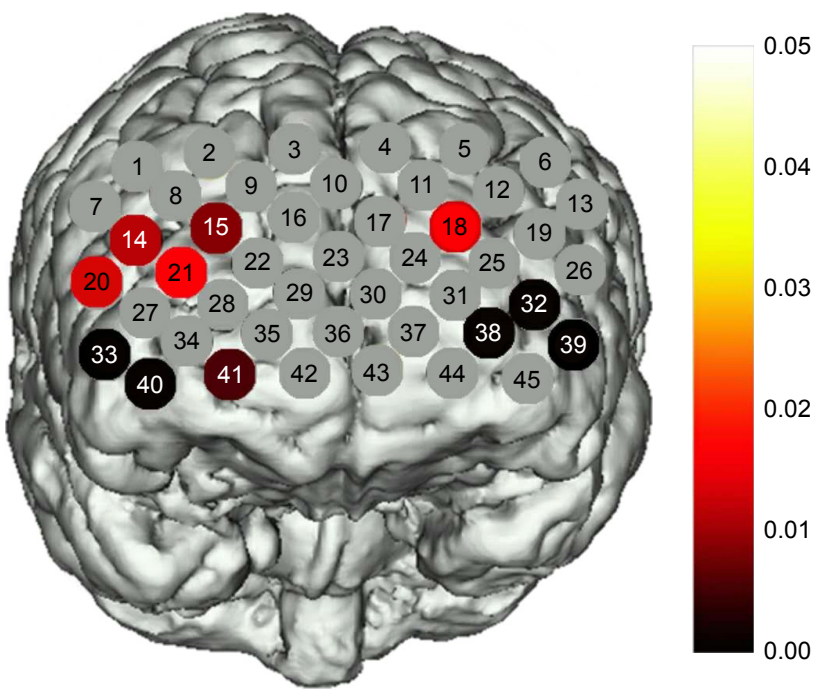

Figure 3 A map showing statistically significant lower oxy-Hb changes in BDp patients compared with the controls during the VFT.

Notes: Channels in color show significantly lower oxy-Hb changes during the VFT in BDp compared with HC (FDR-corrected $P<0.2)$. Channels in gray show FDRcorrected $P$-values $>0.2$.

Abbreviations: $\mathrm{BDp}$, bipolar disorder patients presence of psychotic symptoms; $\mathrm{FDR}$, false discovery rate; $\mathrm{HC}$, healthy controls; oxy-Hb, oxygenated hemoglobin; VFT, verbal fluency test. 

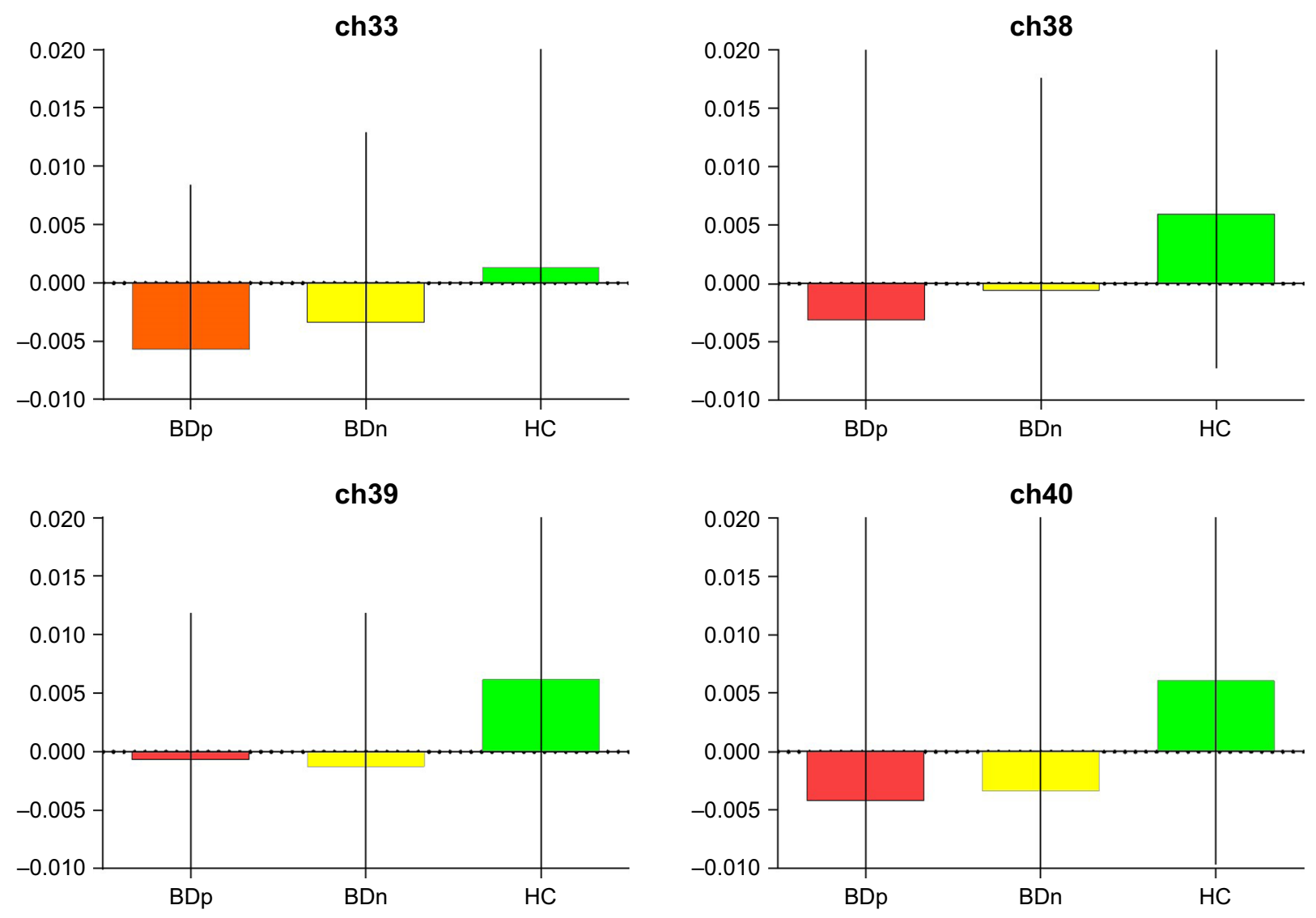

Figure 4 Histogram of the average oxy-Hb concentrations changes in the four channels.

Note: Both BDP and BDn groups showed significantly reduced activation in the four channels than $H C$ (FDR-corrected $P<0.2)$.

Abbreviations: BDp, bipolar disorder patients presence of psychotic symptoms; BDn, bipolar disorder patients absence of psychotic symptoms; FDR, false discovery rate; $\mathrm{HC}$, healthy controls; oxy-Hb, oxygenated hemoglobin.

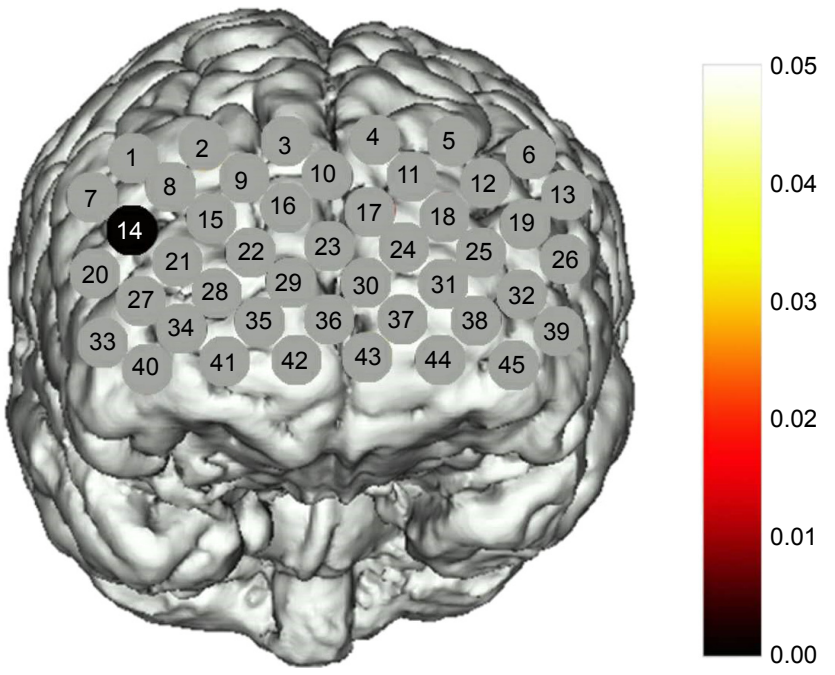

Figure 5 A map showing statistically significant lower oxy-Hb changes in BDp patients compared with BDn during the VFT.

Notes: Channels in color show significantly lower oxy-Hb changes during the VFT in $\mathrm{BDp}$ compared with $\mathrm{BDn}$ (FDR-corrected $P<0.2)$. Channels in gray show FDRcorrected $P$-values $>0.2$.

Abbreviations: $\mathrm{BDp}$, bipolar disorder patients presence of psychotic symptoms; BDn, bipolar disorder patients absence of psychotic symptoms; FDR, false discovery rate; oxy-Hb, oxygenated hemoglobin; VFT, verbal fluency test.

\section{Correlation between NIRS activation and clinical assessments}

In the BDp group, we found a significant negative correlation between the oxy-Hb changes and the BPRS scores in one channel (Ch 14; r: -0.784 ; FDR-corrected $P<0.2$ ), which was located approximately in the right DLPFC region (Figure 6). However, no significant correlation was observed between the changes in mean oxy-Hb and the HAMD and YMRS scores (FDR-corrected $P>0.2$ ) in channels tested in the BDp group.

In the $\mathrm{BDn}$ and $\mathrm{HC}$ groups, there was no significant correlation between the changes in mean oxy- $\mathrm{Hb}$ and the HAMD, YMRS, and BPRS scores (FDR-corrected $P>0.2$ ) in the channels tested.

\section{Discussion}

In the present study, we used VFT as a cognitive task for the exploration of cognitive performance in patients diagnosed with $\mathrm{BD}$, with or without psychotic symptoms, and in a 


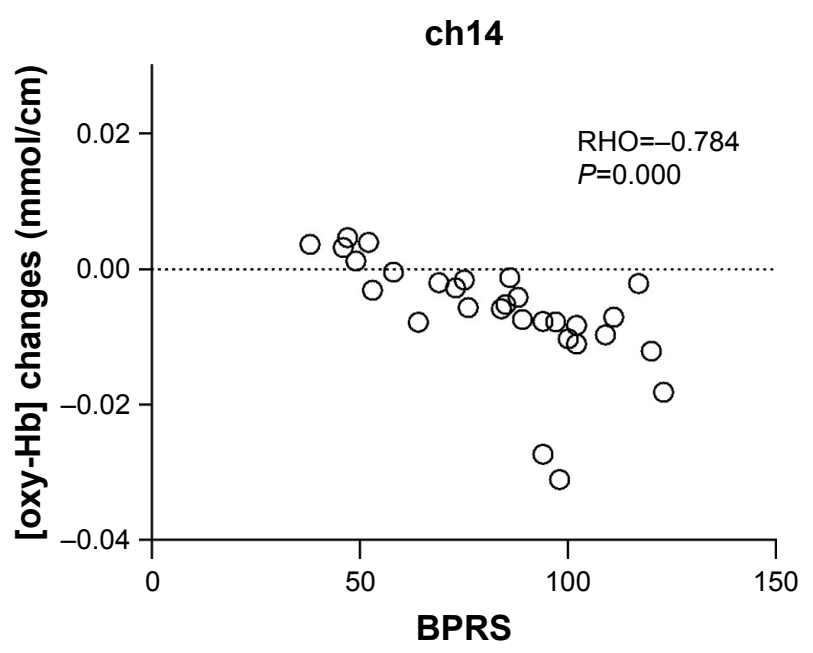

Figure 6 Scatter plot of the oxy-Hb changes in channel 14 changing with BPRS scores.

Note: oxy-Hb changes of channel 14 were negatively correlated with BPRS scores. Abbreviations: BPRS, Brief Psychiatric Rating Scale; oxy-Hb, oxygenated hemoglobin.

control group. We also used NIRS imaging to explore oxy- $\mathrm{Hb}$ changes in the PFC during the performance of these VFTs.

Both the $\mathrm{BD}$ groups were determined to perform significantly worse in the VFT, and a lower activation of oxy- $\mathrm{Hb}$ changes in the bilateral VLPFC was detected in these individuals when they were compared with the $\mathrm{HC}$ group. Within the BD groups, no significant difference was found in the cognition performance. However, the prefrontal activation changes in $\mathrm{BDp}$ patients were determined to be significantly lower than that in BDn patients in the right DLPFC. Activation of oxy-Hb in the right DLPFC was determined to be negatively correlated with psychotic symptoms in BDp patients.

To our knowledge, this is the first report of a study using NIRS technology that has involved the identification of the relationship between VFT-related hemodynamic responses in the prefrontal cortical regions and psychotic symptoms in patients with BD. NIRS may assist in the understanding of the neural basis of psychotic symptoms in patients with BD.

\section{Impaired executive function in BD group}

In comparison to the $\mathrm{HC}$ group, both $\mathrm{BD}$ groups performed significantly worse in terms of generating fewer words. The VFT is a psychological test for the investigation of executive control and cognitive function. These results may imply that there is impairment of cognitive and executive function in BD patients. The decreased number of words recalled during the VFT may result from the deterioration of semantic information, according to the storage-deficit hypothesis, or from dysfunction of the frontal lobe relating to the ability to retrieve a series of nouns. ${ }^{48}$ Previous studies reported in the literature show significant impairments in VFT, supporting our current findings. ${ }^{49-51}$ Aprahamian et al ${ }^{49}$ reported data derived from a group of 86 patients with $\mathrm{BD}$, where they showed worse performances on the VFT. Meesters et $\mathrm{al}^{50}$ and Radanovic et $\mathrm{al}^{51}$ also identified evidence of performance deficits in individuals with $\mathrm{BD}$ and also suggested that these impairments existed in specific cognitive domains, particularly executive functionality. Such impairments of executive function can lead to the destruction of a patient's daily life..$^{52}$ They could mean a reduced ability to learn, ability to be independent, and to make social connections. Previous studies have also failed to show significant cognitive impairment in BD patients. ${ }^{9,53}$ The discrepancies in the literature could be due to the different moods of the patients studied, differences in the courses and types of the BD involved, and differences in the cognitive tasks employed by researchers. The negative result, in terms of the differences in the accuracy (\%) of correct words, may have been due to the simple test used and the timings used, but this remains unclear. ${ }^{54}$ In the present study, the time allowed for each category of cognitive task was 30 seconds. It was found that in the first time interval there was a rush of words produced, and that the numbers decreased in subsequent time intervals. ${ }^{55}$ Increased time to implement the relevant search process is the reciprocal of the fact that as the time on task increases, searching becomes harder and achieving the target words becomes less frequent. ${ }^{56}$

The number of words generated was no different between the BDp and BDn groups. So, the extent of the cognitive impairment of mainly executive function in the BD groups was similar. This finding demonstrates that psychotic symptoms may have a minimal impact on the cognitive performance in VFT. However, there are also studies ${ }^{16}$ in which it was found that BD patients with a history of psychotic episodes were significantly cognitively impaired in terms of executive functioning performance and spatial working memory compared with BDn patients. Although semantic fluency was also applied to examine executive function, the effect of different languages such as Chinese or English on performance in the VFT remains unclear. Nevertheless, here, we observed different degrees of PFC activation.

\section{Hypoactivation of the bilateral VLPFC in BD groups}

In the present study, lower oxy-Hb activation, compared with the HC group, was observed in the bilateral VLPFC (channels 36,39 , and 41) during the VFT in BD patients with or without 
psychotic symptoms. Previous functional neuroimaging studies ${ }^{57-59}$ have consistently found decreased activation in the VLPFC during cognitive tasks in BD patients when they are compared with $\mathrm{HC}$ subjects. The prefrontal hypoactivation during the task period may imply that the BD patients failed to obtain a corresponding increase in blood supply to compensate for the oxygen consumed, which is crucial for optimal neuronal activity. The VLPFC ${ }^{60}$ plays an important role in the integration of emotional information and the regulation of emotional responses, and hypoactivity in this area suggests that BD patients in a depressive episode, whether with or without psychotic symptoms, have malfunctions in their emotional regulation and integration.

\section{The function of the right DLPFC and psychotic symptoms}

We observed a lower activation of channels 14, 15, 18, 20, 21, 32, 33, 38-41, located the VLPFC and DLPFC, in BD patients in a depressive episode with psychotic symptoms when this was compared with the HC group. A lower activation in channel 14 of the right DLPFC in the BDp group when compared with the BDn group was also observed. In addition, BDp showed a negative correlation with oxy-Hb changes in channel 14, near the region of right DLPFC. This may represent neural vulnerability in terms of the severity of psychotic symptoms. The lower oxy- $\mathrm{Hb}$ activation in the right DLPFC showed a trend to accentuate gradually with an increase in the severity of psychotic symptoms.

Previous studies have shown a reduced connectivity of the DLPFC and other brain regions (amygdala) in individuals with BD with psychotic symptoms. ${ }^{12}$ Reduction in the volume of the GM in the DLPFC using morphometric MRI was also identified in bipolar patients with persecutory delusions. ${ }^{10}$ Lower activation of oxy-Hb near the DLPFC area was also demonstrated in a previous study using VFT in Chinesespeaking schizophrenics. ${ }^{61}$ In a systematic review, evidence suggested that hypofunctioning of the DLPFC during executive functioning could be a neural marker of psychosis. ${ }^{62}$ From a clinical point of view, the DLPFC plays an important role in the assessment of psychotic symptoms.

Structural and functional studies suggest that the DLPFC is linked to executive functioning ${ }^{63}$ and that the DLPFC is part of the network, which is hypothesized to be related to the monitoring of introspective mental activity. ${ }^{64}$ Diminished executive functionality and the reduced monitoring of mental activity may result in dysfunction of multiple cognitive and reasoning processes, which could manifest as psychotic symptoms. The DLPFC can avoid irrelevant information and focus on targeted information as the priority, in bottom-up and top-down attention. ${ }^{65}$ Patients with delusional symptoms are unable to distinguish between relevant and irrelevant sensory stimuli, and a hallucination is a perception without a stimulus. Such symptoms could occur due to dysfunction of the DLPFC when it is unable to avoid irrelevant information. BDn patients were asymptomatic at the time of this study. We did not know if they had exhibited psychotic symptoms in the past episode, thus we cannot draw the conclusion that the DLPFC represents the neurobiological basis of psychotic symptoms. It is clear that the DLPFC is related to the presence of psychotic symptoms, so that DLPFC dysfunction is a marker of the state of having psychotic symptoms not a marker of the trait.

BD patients in a depressive episode with psychotic symptoms showed decreased activation of the VLPFC and DLPFC, while BD patients in a depressive episode without psychotic symptoms only showed decreased activation of the VLPFC. So, it would be reasonable to speculate that different areas of prefrontal cortical functionality may represent different clinical symptoms such as mood instability and psychosis. The results presented here may suggest that dysfunction of the VLPFC is particularly associated with the symptoms of mood change and that dysfunction of the DLPFC is associated with psychotic symptoms in $\mathrm{BD}$ patients during a depressive episode.

\section{Limitations}

This study should be interpreted according to its limitations. Firstly, NIRS measures only the brain's surface and so detects only the relative concentration of oxy- $\mathrm{Hb}$. As a result, activity in deep brain structures cannot be measured. Secondly, in the present study, participating BDp patients were exhibiting symptoms at the time of examination, and we did not take account of retrospective symptoms. In addition, patients with a whole range of psychotic symptoms were enrolled in this research. Thirdly, in the present study, cognitive impairment refers to cognitive performance on a specific and simple test, not cognition generally. Fourthly, the present study focused on BD-I patients during the depressive phase, while psychotic symptoms are more common in manic episodes and BD-I and BD-II patients are known to be different in terms of brain function. Further studies into the subtypes of BD with psychotic symptoms are required to confirm the effects of psychotic symptoms on BD patients.

\section{Conclusion}

Although BD patients, during a depressive episode, exhibited significantly worse performances in the VFT compared 
with $\mathrm{HC}$ subjects, the cognitive performance in the VFT was not different in BD patients with and without psychotic symptoms in the depressive episode. Using NIRS, BD patients in the depressive episode exhibited hypoactivation of the bilateral VLPFC during cognitive tasks. Furthermore, prefrontal activation of oxy-Hb changes in the right DLPFC was significantly lower in BDp patients in the depressive episode compared with BDn patients in the depressive episode. Hypoactivity of the right DLPFC was associated with psychotic symptoms in BDp patients. These findings provide novel evidence of PFC dysfunction in BD patients with psychotic features and show that NIRS can be a useful tool for evaluating PFC functioning associated with psychotic characteristics in $\mathrm{BD}$ during a depressive episode.

\section{Acknowledgments}

The authors thank all the participants in this study. This study was supported by the Independent Scientific Research Program of Tsinghua University (No 548105001).

\section{Disclosure}

The authors report no conflicts of interest in this work.

\section{References}

1. Goodwin FJK. Manic-Depressive Illness. Oxford, UK: Oxford University Press; 1990.

2. Keck PE, McElroy SL, Havens JR, et al. Psychosis in bipolar disorder: phenomenology and impact on morbidity and course of illness. Compr Psychiatry. 2003;44(4):263-269.

3. Tohen M, Zarate CA Jr, Hennen J, et al. The McLean-Harvard FirstEpisode Mania Study: prediction of recovery and first recurrence. Am J Psychiatry. 2003;160(12):2099-2107.

4. Coryell W, Leon AC, Turvey C, Akiskal HS, Mueller T, Endicott J. The significance of psychotic features in manic episodes: a report from the NIMH collaborative study. J Affect Disord. 2001;67(1-3):79-88.

5. Goghari VM, Harrow M. Twenty year multi-follow-up of different types of hallucinations in schizophrenia, schizoaffective disorder, bipolar disorder, and depression. Schizophr Res. 2016;176(2-3):371-377.

6. Solomon DA, Leon AC, Coryell WH, et al. Longitudinal course of bipolar I disorder: duration of mood episodes. Arch Gen Psychiatry. 2010;67(4):339-347.

7. Caldieraro MA, Sylvia LG, Dufour S, et al. Clinical correlates of acute bipolar depressive episode with psychosis. J Affect Disord. 2017;217:29-33.

8. Gruber SA, Dahlgren MK, Sagar KA, et al. Decreased cingulate cortex activation during cognitive control processing in bipolar disorder. J Affect Disord. 2017;213:86-95.

9. Nishimura Y, Takahashi K, Ohtani T, Ikeda-Sugita R, Kasai K, Okazaki Y. Dorsolateral prefrontal hemodynamic responses during a verbal fluency task in hypomanic bipolar disorder. Bipolar Disord. 2015;17(2):172-183.

10. Radaelli D, Poletti S, Gorni I, et al. Neural correlates of delusion in bipolar depression. Psychiatry Res. 2014;221(1):1-5.

11. Anticevic A, Savic A, Repovs G, et al. Ventral anterior cingulate connectivity distinguished nonpsychotic bipolar illness from psychotic bipolar disorder and schizophrenia. Schizophr Bull. 2015;41(1):133-143.

12. Anticevic A, Brumbaugh MS, Winkler AM, et al. Global prefrontal and fronto-amygdala dysconnectivity in bipolar I disorder with psychosis history. Biol Psychiatry. 2013;73(6):565-573.
13. Martinez-Aran A, Torrent C, Tabares-Seisdedos R, et al. Neurocognitive impairment in bipolar patients with and without history of psychosis. J Clin Psychiatry. 2008;69(2):233-239.

14. Torres IJ, Boudreau VG, Yatham LN. Neuropsychological functioning in euthymic bipolar disorder: a meta-analysis. Acta Psychiatr Scand Suppl. 2007;116(434):17-26.

15. Bonnín CM, Martínez-Arán A, Torrent C, et al. Clinical and neurocognitive predictors of functional outcome in bipolar euthymic patients: a long-term, follow-up study. J Affect Disord. 2010;121(1-2):156-160.

16. Glahn DC, Bearden CE, Barguil M, et al. The neurocognitive signature of psychotic bipolar disorder. Biol Psychiatry. 2007;62(8):910-916.

17. Simonsen C, Sundet K, Vaskinn A, et al. Neurocognitive dysfunction in bipolar and schizophrenia spectrum disorders depends on history of psychosis rather than diagnostic group. Schizophr Bull. 2011;37(1): 73-83.

18. Selva G, Salazar J, Balanzá-Martínez V, et al. Bipolar I patients with and without a history of psychotic symptoms: do they differ in their cognitive functioning? J Psychiatr Res. 2007;41(3-4):265-272.

19. Lahera G, Montes JM, Benito A, et al. Theory of mind deficit in bipolar disorder: is it related to a previous history of psychotic symptoms? Psychiatry Res. 2008;161(3):309-317.

20. Schecklmann M, Dresler T, Beck S, et al. Reduced prefrontal oxygenation during object and spatial visual working memory in unpolar and bipolar depression. Psychiatry Res. 2011;194(3):378-384.

21. Takizawa R, Fukuda M, Kawasaki S, et al. Neuroimaging-aided differential diagnosis of the depressive state. Neuroimage. 2014;85(Pt 1): 498-507.

22. Matsubara T, Matsuo K, Nakashima M, et al. Prefrontal activation in response to emotional words in patients with bipolar disorder and major depressive disorder. Neuroimage. 2014;85(Pt 1):489-497.

23. Kubota Y, Toichi M, Shimizu M, et al. Altered prefrontal lobe oxygenation in bipolar disorder: a study by near-infrared spectroscopy. Psychol Med. 2009;39(8):1265-1275.

24. Kameyama M, Fukuda M, Yamagishi Y, et al. Frontal lobe function in bipolar disorder: a multichannel near-infrared spectroscopy study. Neuroimage. 2006;29(1):172-184.

25. Altamura AC, Buoli M, Caldiroli A, et al. Misdiagnosis, duration of untreated illness (DUI) and outcome in bipolar patients with psychotic symptoms: a naturalistic study. J Affect Disord. 2015;182:70-75.

26. Frankland A, Cerrillo E, Hadzi-Pavlovic D, et al. Comparing the phenomenology of depressive episodes in bipolar I and II disorder and major depressive disorder within bipolar disorder pedigrees. $J$ Clin Psychiatry. 2015;76(1):32-38.

27. Fountoulakis KN, Young A, Yatham L, et al. The International College of Neuropsychopharmacology (CINP) Treatment Guidelines for Bipolar Disorder in Adults (CINP-BD-2017), Part 1: Background and Methods of the Development of Guidelines. Int J Neuropsychopharmacol. 2016;20(2):98-120

28. Dell'Osso L, Pini S, Tundo A, Sarno N, Musetti L, Cassano GB. Clinical characteristics of mania, mixed mania, and bipolar depression with psychotic features. Compr Psychiatry. 2000;41(4):242-247.

29. Merikangas KR, Akiskal HS, Angst J, et al. Lifetime and 12-month prevalence of bipolar spectrum disorder in the National Comorbidity Survey replication. Arch Gen Psychiatry. 2007;64(5):543-552.

30. Krishnan KR. Psychiatric and medical comorbidities of bipolar disorder. Psychosom Med. 2005;67(1):1-8.

31. Hassel S, Almeida JR, Frank E, et al. Prefrontal cortical and striatal activity to happy and fear faces in bipolar disorder is associated with comorbid substance abuse and eating disorder. J Affect Disord. 2009;118(1-3):19-27.

32. Altamura AC, Delvecchio G, Marotta G, et al. Structural and metabolic differentiation between bipolar disorder with psychosis and substanceinduced psychosis: An integrated MRI/PET study. Eur Psychiatry. 2017;41:85-94.

33. Yokoyama C, Kaiya H, Kumano H, et al. Dysfunction of ventrolateral prefrontal cortex underlying social anxiety disorder: a multi-channel NIRS study. Neuroimage Clin. 2015;8:455-461. 
34. Hirosawa R, Narumoto J, Sakai Y, et al. Reduced dorsolateral prefrontal cortical hemodynamic response in adult obsessive-compulsive disorder as measured by near-infrared spectroscopy during the verbal fluency task. Neuropsychiatr Dis Treat. 2013;9:955-962.

35. Okada K, Ota T, Iida J, Kishimoto N, Kishimoto T. Lower prefrontal activity in adults with obsessive-compulsive disorder as measured by near-infrared spectroscopy. Prog Neuropsychopharmacol Biol Psychiatry. 2013;43:7-13.

36. C Schudlo L, Chau T. Towards a ternary NIRS-BCI: single-trial classification of verbal fluency task, Stroop task and unconstrained rest. J Neural Eng. 2015;12(6):066008.

37. Kahlaoui K, Di Sante G, Barbeau J, et al. Contribution of NIRS to the study of prefrontal cortex for verbal fluency in aging. Brain Lang. 2012;121(2):164-173.

38. Schwartz S, Baldo J. Distinct patterns of word retrieval in right and left frontal lobe patients: a multidimensional perspective. Neuropsychologia. 2001;39(11):1209-1217.

39. Romano R, Defazio G. Neurocognitive assessment in adult-onset primary cranial cervical dystonia - response to the letter. Parkinsonism Relat Disord. 2014;20(8):941.

40. Kakimoto Y, Nishimura Y, Hara N, Okada M, Tanii H, Okazaki Y. Intrasubject reproducibility of prefrontal cortex activities during a verbal fluency task over two repeated sessions using multi-channel near-infrared spectroscopy. Psychiatry Clin Neurosci. 2009;63(4):491-499.

41. Okamoto M, Dan H, Sakamoto K, et al. Three-dimensional probabilistic anatomical cranio-cerebral correlation via the international 10-20 system oriented for transcranial functional brain mapping. Neuroimage. 2004;21(1):99-111.

42. Hamilton M. A rating scale for depression. J Neurol Neurosurg Psychiatry. 1960;23:56-62.

43. Young RC, Biggs JT, Ziegler VE, Meyer DA. A rating scale for mania: reliability, validity and sensitivity. Br J Psychiatry. 1978;133:429-435.

44. Ventura J, Green MF, Shaner A, Liberman RP. Training and quality assurance with the Brief Psychiatric Rating Scale: "The drift busters." Int J Methods Psychiatric Res. 1993;3(4):221-244.

45. Huppert TJ, Hoge RD, Diamond SG, Franceschini MA, Boas DA. A temporal comparison of BOLD, ASL, and NIRS hemodynamic responses to motor stimuli in adult humans. Neuroimage. 2006;29(2):368-382.

46. Hori H, Ozeki Y, Terada S, Kunugi H. Functional near-infrared spectroscopy reveals altered hemispheric laterality in relation to schizotypy during verbal fluency task. Prog Neuropsychopharmacol Biol Psychiatry. 2008;32(8):1944-1951.

47. Kono T, Matsuo K, Tsunashima K, et al. Multiple-time replicability of near-infrared spectroscopy recording during prefrontal activation task in healthy men. Neurosci Res. 2007;57(4):504-512.

48. Bozikas VP, Kosmidis MH, Karavatos A. Disproportionate impairment in semantic verbal fluency in schizophrenia: differential deficit in clustering. Schizophr Res. 2005;74(1):51-59.

49. Aprahamian I, Ladeira RB, Diniz BS, Forlenza OV, Nunes PV. Cognitive impairment in euthymic older adults with bipolar disorder: a controlled study using cognitive screening tests. Am J Geriatr Psychiatry. 2014;22(4):389-397.
50. Meesters PD, Schouws S, Stek M, et al. Cognitive impairment in late life schizophrenia and bipolar I disorder. Int J Geriatr Psychiatry. 2013;28(1):82-90.

51. Radanovic M, Nunes PV, Forlenza OV, Braga Ladeira R, Gattaz WF. Cognitive-linguistic deficits in euthymic elderly patients with bipolar disorder. J Affect Disord. 2013;150(2):691-694.

52. Goel V, Grafman J, Tajik J, Gana S, Danto D. A study of the performance of patients with frontal lobe lesions in a financial planning task. Brain. 1997;120 (Pt 10):1805-1822.

53. Barrett SL, Kelly C, Bell R, King DJ. Gender influences the detection of spatial working memory deficits in bipolar disorder. Bipolar Disord. 2008;10(5):647-654.

54. Noda T, Yoshida S, Matsuda T, et al. Frontal and right temporal activations correlate negatively with depression severity during verbal fluency task: a multi-channel near-infrared spectroscopy study. J Psychiatr Res. 2012;46(7):905-912.

55. Crowe SF. Decrease in performance on the verbal fluency test as a function of time: evaluation in a young healthy sample. J Clin Exp Neuropsychol. 1998;20(3):391-401.

56. Warburton E, Wise RJ, Price CJ, et al. Noun and verb retrieval by normal subjects. Studies with PET. Brain. 1996;119 (Pt 1):159-179.

57. Belmaker RH. The bipolar brain: integrating neuroimaging and genetics. Am J Psychiatry. 2013;170(1):127-128.

58. Blumberg HP, Leung HC, Skudlarski P, et al. A functional magnetic resonance imaging study of bipolar disorder: state- and trait-related dysfunction in ventral prefrontal cortices. Arch Gen Psychiatry. 2003; 60(6):601-609

59. Strakowski SM, Adler CM, Holland SK, Mills NP, DelBello MP, Eliassen JC. Abnormal FMRI brain activation in euthymic bipolar disorder patients during a counting Stroop interference task. Am J Psychiatry. 2005;162(9):1697-1705.

60. Ochsner KN, Gross JJ. The cognitive control of emotion. Trends Cogn Sci. 2005;9(5):242-249.

61. Quan W, Wu T, Li Z, Wang Y, Dong W, Lv B. Reduced prefrontal activation during a verbal fluency task in Chinese-speaking patients with schizophrenia as measured by near-infrared spectroscopy. Prog Neuropsychopharmacol Biol Psychiatry. 2015;58:51-58.

62. Fusar-Poli P, Perez J, Broome M, et al. Neurofunctional correlates of vulnerability to psychosis: a systematic review and meta-analysis. Neurosci Biobehav Rev. 2007;31(4):465-484.

63. Miller BL, Cummings JL. The Human Frontal Lobes: Functions and Disorders. Oxfordshire, UK: Taylor \& Francis Ltd; 2007.

64. Gusnard DA, Raichle ME, Raichle ME. Searching for a baseline: functional imaging and the resting human brain. Nat Rev Neurosci. 2001;2(10):685-694.

65. Valet M, Gündel H, Sprenger T, et al. Patients with pain disorder show gray-matter loss in pain-processing structures: a voxel-based morphometric study. Psychosom Med. 2009;71(1):49-56.
Neuropsychiatric Disease and Treatment

\section{Publish your work in this journal}

Neuropsychiatric Disease and Treatment is an international, peerreviewed journal of clinical therapeutics and pharmacology focusing on concise rapid reporting of clinical or pre-clinical studies on a range of neuropsychiatric and neurological disorders. This journal is indexed on PubMed Central, the 'PsycINFO' database and CAS,
Dovepress

and is the official journal of The International Neuropsychiatric Association (INA). The manuscript management system is completely online and includes a very quick and fair peer-review system, which is all easy to use. Visit http://www.dovepress.com/testimonials.php to read real quotes from published authors. 